\title{
DESENVOLVIMENTO DE MÉTODOS DE AMOSTRAGEM E ANÁLISES DE COMPOSTOS SULFURADOS EM GAS NATURAL
}

\author{
L. R. F. COELHO ${ }^{1}$, M. F. AGUIAR ${ }^{1}$ e G. L. V. COELHO ${ }^{1}$ \\ ${ }^{1}$ Universidade Federal Rural do Rio de Janeiro, Departamento de Engenharia Química \\ E-mail para contato: coelho@ufrrj.br
}

RESUMO - As baixas concentrações de espécies de enxofre, compostos indesejáveis, frequentemente encontrados em correntes de gás natural, proporcionam dificuldades na amostragem e a análises destes. Para o desenvolvimento de processos de separação e purificação de gás natural faz-se necessário o conhecimento e/ou aperfeiçoamento de métodos de amostragem e de análise. Com a finalidade de avaliar e discutir estes problemas relacionados à amostragem de compostos sulfurados, utilizou-se um gás natural sintético com nove compostos de enxofre de concentrações conhecidas que foi analisado em cromatografia gasosa e espectrometria de massas através de três diferentes métodos de amostragem usando um detector FID.

\section{INTRODUÇÃO}

Gás Natural é um combustível fóssil encontrado em rochas porosas no subsolo terrestre ou marítimo. Sua composição consiste em uma mistura de hidrocarbonetos, onde seu principal componente é o metano (JESUS, C. S. et al. , 2012). Em comparação com outros combustíveis fósseis, como o petróleo e carvão mineral, o gás natural apresenta menos riscos ao meio ambiente, pois apresenta baixos teores de contaminantes, como nitrogênio $\left(\mathrm{N}_{2}\right)$, dióxido de carbono $\left(\mathrm{CO}_{2}\right)$ e compostos sulfurados, principalmente sob a forma de ácido sulfídrico $\left(\mathrm{H}_{2} \mathrm{~S}\right)$ (SHIMEKIT \& MUKHTAR, 2012). Outros compostos indesejáveis contendo enxofre são frequentemente encontrados em correntes de gás natural na forma de mercaptanos, sulfetos, dissulfetos ou compostos heterocíclicos, presentes em concentrações variáveis, influenciadas pela origem do petróleo e por processos de refinaria (DIAS, 2008). A presença destes compostos pode provocar corrosão, envenenamento de catalisadores, ou produzir odores desagradáveis (GRAS et al., 2009).

No desenvolvimento de processos de separação, dificuldades são encontradas na amostragem e análises destes compostos, frutos de suas volatilidades à temperatura ambiente e por alguns serem solúveis em água. Como são encontrados geralmente em pequenas concentrações em matrizes complexas na faixa de $\mathrm{ng} / \mathrm{m}^{-3}$ a $\mu \mathrm{g} / \mathrm{m}^{-3}$, a fim de melhorar a sensibilidade do método e/ou o isolamento do composto de interesse, reduzindo ou eliminando possíveis interferências, uma etapa de pré- concentração é feita durante a amostragem (CRUZ \& CAMPOS, 2008).

Em virtude da volatilidade da maioria dos contaminantes de enxofre, o método analítico mais utilizado para determinar esses compostos sulfurados é a Cromatografia Gasosa (DIAS, 2008). Neste método físico-químico, um fator crítico é a determinação da resolução necessária para ocorrer a separação (AUGUSTO, 2011). Devido aos problemas que são encontradas na quantificação destes no CG, foram então desenvolvidos métodos de detecção 
sensíveis a esses compostos, tais como detector fotométrico de chama (FPD), detector fotométrico de chama-pulsada (P-FPD), quimiluminescência Dual-plasma (DP-DF) ou espectrometria de massa (MS) entre outros (GRAS et al., 2009).

Amostras provenientes de matrizes complexas, como o caso do gás natural, os contaminantes encontram-se em quantidades traços. Nesses casos, a coeluição desses compostos, que estão presentes em quantidades mínimas, com os constituintes predominantes na amostra, como o metano, gera uma relação sinal-ruído muito grande, aumentando o erro na quantificação desses contaminantes de interesse. O emprego de detectores específicos e colunas cromatográficas adequadas para compostos voláteis podem auxiliar, mas a identificação de compostos voláteis em matrizes complexas continua sendo um desafio (MACHADO, 2011)

Outras soluções analíticas, como os moduladores criogênicos baseados em jatos frios estão sendo cada vez mais empregados. Nestes moduladores, o resfriamento é feito através de jatos de $\mathrm{CO}_{2}$ ou de $\mathrm{N}_{2}$ líquidos diretamente lançados sobre a coluna, permitindo a separação de analitos altamente voláteis (PEDROSO et al., 2009). Embora essas soluções analíticas sejam adequadas, são técnicas de restrita implantação e alto custo. Devido às dificuldades citadas, o objetivo desse trabalho foi avaliar diferentes técnicas de amostragem em um sistema cromatográfico que utiliza um detector de ionização de chama, a fim de obter uma metodologia eficiente e de baixo custo na identificação de compostos traços sulfurados e que será usado no monitoramento do processo de separação em desenvolvimento para purificação de gas natural.

\section{MATERIAIS E MÉTODOS}

A amostra analisada foi confeccionada pela empresa White Martins cuja composição se encontra na Tabela 1.

Tabela 1: Composição da Amostra de Gás Natural

\begin{tabular}{lcl}
\hline Analitos & Conc.(ppmv) & Conc.(ppmv S) \\
DMS (Dimetilsulfito) & 47.9 & 1.6 \\
COS(sulfureto de carbonilo) $+\mathrm{SO}_{2}$ & 49.2 & 1.7 \\
t-BM(Tertbutilmercaptano) & 50.6 & 1.7 \\
s-BM(Sec-butilmercaptano) & 50.4 & 1.7 \\
i-PM(Isopropilmercaptano) & 49.8 & 1.7 \\
MM(Metilmercaptano) & 47.9 & 1.6 \\
EM(Etilmercaptano) & 50.7 & 1.7 \\
THT(Tetrahidrotiofeno) & 198.0 & 6.7 \\
MES(metiletilsulfito) & 46.8 & 1.6 \\
Metano & restante & restante \\
\hline
\end{tabular}

A identificação dos compostos de enxofre foi realizada em um cromatógrafo a gás aclopado a um espectrômetro de massas (GSMS-QP 2010 SHIMADZU) equipado com uma coluna capilar SPB-1 $60 \mathrm{~m}$ x 0.32mm x $5 \mu \mathrm{m}$ (SUPELCO). Foram recolhidos de uma Sample Bag, $500 \mu \mathrm{L}$ da amostra de gás natural e injetados no modo splitless. Com base em testes preliminares foi determinada a melhor condição cromatográfica para a identificação dos compostos de interesse. Sendo assim, a temperatura inicial do forno foi de $35^{\circ} \mathrm{C}$ por 5 
minutos, em seguida a temperatura foi elevada a $65^{\circ} \mathrm{C}$ com uma rampa de aquecimento de $1^{\circ} \mathrm{C} / \mathrm{min}$, na qual permaneceu por 1 minuto. Posteriormente, essa temperatura foi elevada a $160^{\circ} \mathrm{C}$ com uma taxa de aquecimento de $15^{\circ} \mathrm{C} / \mathrm{min}$, permanecendo nessa temperatura final por 5 minutos. $\mathrm{O}$ injetor foi mantido a $150^{\circ} \mathrm{C}$, a temperatura da fonte de íons foi de $150^{\circ} \mathrm{C}$ e da interface de transferência $180^{\circ} \mathrm{C}$. A faixa de massas analisadas foi entre 10 a $150 \mathrm{~m} / \mathrm{z}$. A identificação dos compostos foi feita pela comparação das massas de cada substância com as massas da biblioteca utilizada (NIST 05). Foram considerados apenas os componentes que apresentaram similaridade superior a $90 \%$ em relação dos fragmentos da biblioteca utilizada.

Foram utilizados 3 diferentes métodos de amostragem para análise do gás natural no GC HP-5890: SPME, Injeção direta e Sample bag. As condições cromatográficas foram as mesmas descritas no método de identificação dos compostos, com a temperatura do detector de ionização de chamas (FID) mantido à $250^{\circ} \mathrm{C}$.

A injeção direta foi realizada através de uma válvula de 6 vias conectada ao cromatógrafo. Já para injeção indireta foi utilizado uma Sample bag Tedlar de 1L para armazenamento da amostra, em seguida foram injetados $500 \mu \mathrm{L}$ no GC.

No método de amostragem por SPME, foi utilizada uma fibra de CAR/PDMS submetida à uma temperatura de $22^{\circ} \mathrm{C}$ e tempo de extração de 20 min na Sample Bag, posteriormente introduzida no injetor do cromatógrafo e dessorvida à $150^{\circ} \mathrm{C}$ por $2 \mathrm{~min}$. A fibra foi condicionada à $250^{\circ} \mathrm{C}$ por $10 \mathrm{~min}$ como apresentado por Nielsen e Jonsson (2002).

\section{RESULTADOS E DISCUSSÕES}

A Figura 1 representa o cromatograma do gás natural, analisado por um GC/MS onde foi possível identificar sete dos dez compostos presentes na amostra. Todos os compostos identificados apresentaram similaridade superior a 90\% em relação aos fragmentogramas da biblioteca utilizada (NIST 05). Os compostos sulfurados então presentes em quantidades traços no gás natural, sendo o metano o componente principal, representando mais de $90 \%$ do volume da amostra, portanto foi necessário iniciar a detecção no GC/MS após o tempo de retenção do mesmo, pois sua alta concentração satura o detector acarretando possível encobrimento dos compostos com tempo de retenção próximo do mesmo.

Figura 1: Espectrograma da amostra de gás natural armazenada em uma Sample Bag. (1) etilmercaptana, (2) dimetisulfeto, (3) isopropilmercaptana, (4) terc-butilmercaptna, (5) metiletilsulfeto, (6) sec-butilmercaptana, (7) tetrahidrotiofeno.

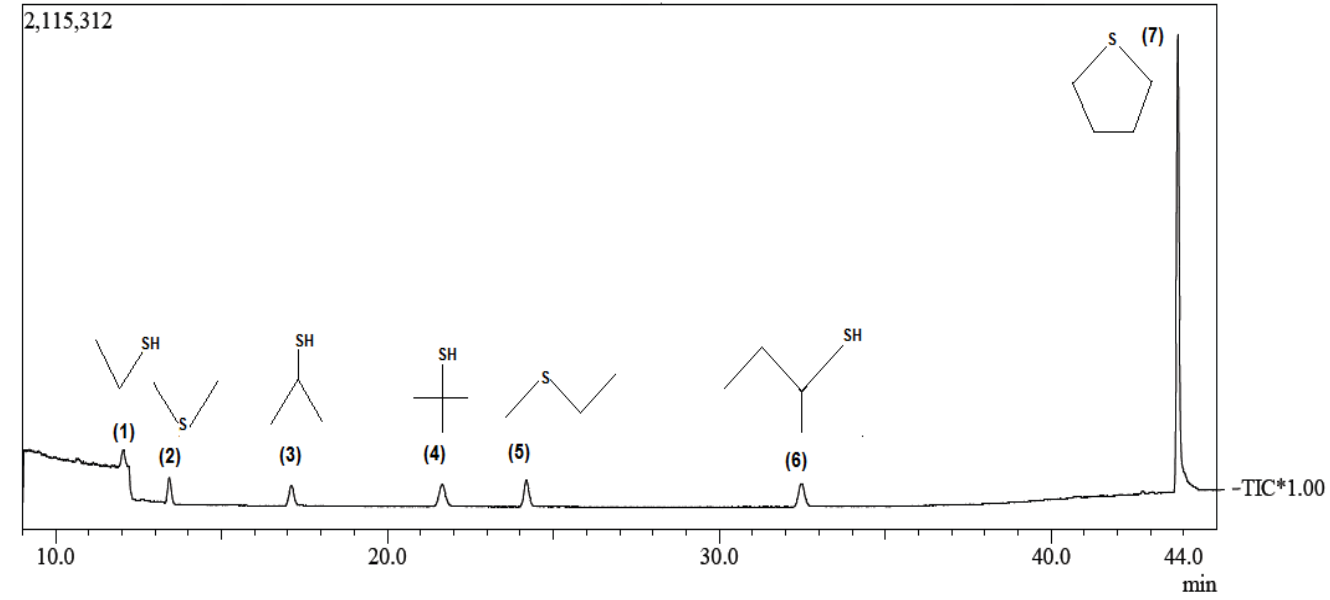


As condições experimentais das análises no CG apresentaram bons resultados na detecção dos compostos, pois para os diferentes métodos de amostragem os sete compostos identificados no GC/MS resultaram em tempos de retenção próximos, como pode ser observado na Figura 2.

Figura 2: Cromatograma dos compostos sulfurados presente no Gás Natural (a) Amostragem por injeção indireta, (b) Amostragem por injeção direta, (c) SPME - CAR/PDMS

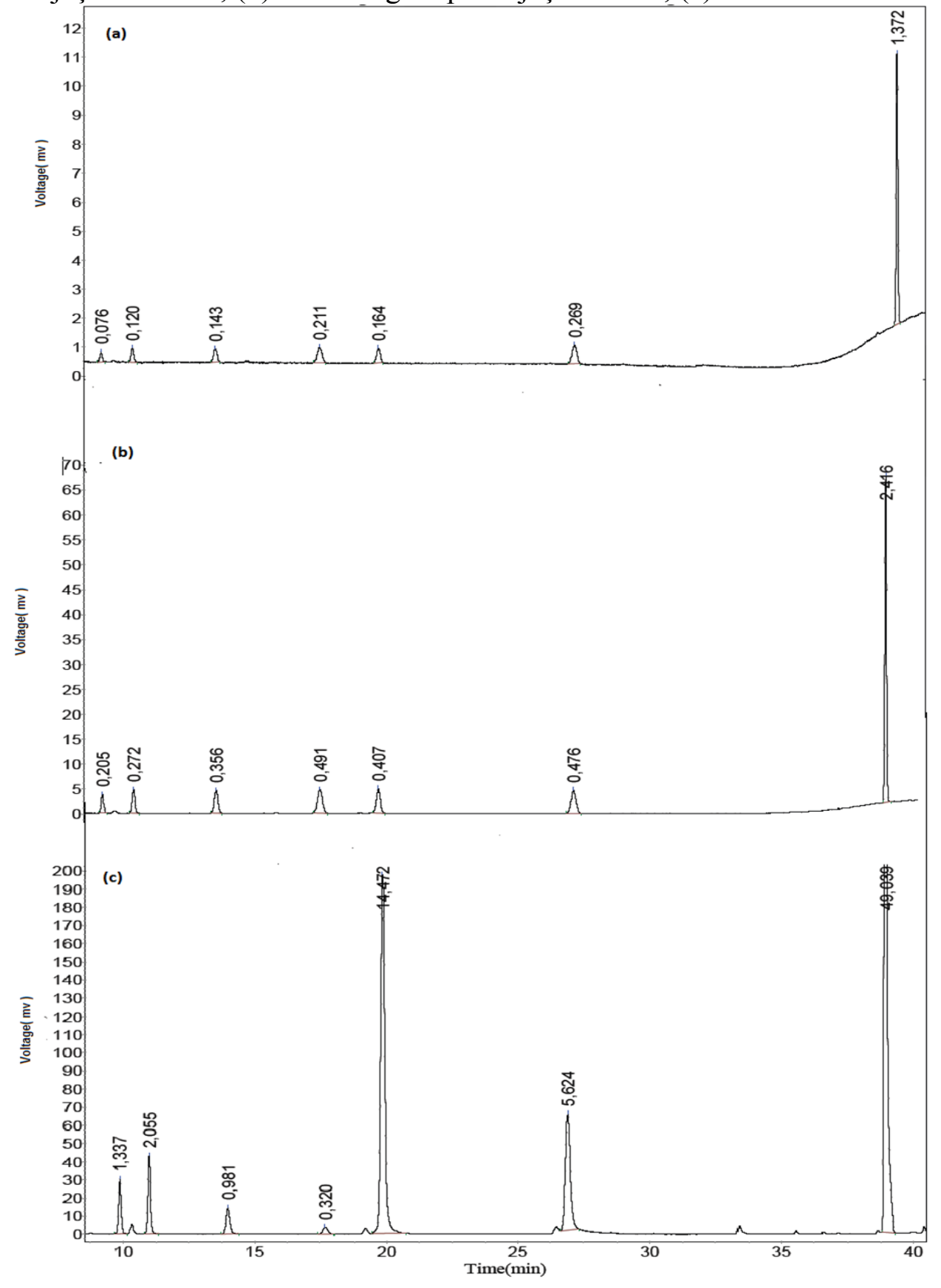

Devido à baixa concentração de espécies de enxofre (nível ppb ou ppt), a préconcentração na etapa de amostragem é importante, pois esse processo é responsável pelo 
aumento da relação sinal-ruído, ou seja, um aumento na relação entre os componentes traços de interesse e os compostos com altas concentrações da matriz, influenciando no limite de detecção analítico, reduzindo o efeito de matriz e podendo melhorar a exatidão dos resultados (CRUZ \& CAMPOS, 2008). Isso foi observado na Figura 3, onde o método de amostragem por SPME apresentou maiores percentagens de área em relação aos outros dois métodos.

O recobrimento da fibra de SPME (Micro Extração em Fase Sólida) é escolhido em função do analito a ser determinado, e a natureza da mesma influencia fortemente na efetividade da técnica (KAWASE, 2013). A seletividade da fibra explica a maior percentagem área para alguns compostos sulfurados como o metiletilsulfeto e o tetrahidrotiofeno, observada na Figura 2(c). Já o terc-butilmercaptana não apresentou a mesma afinidade com a fibra.

As samples bags Tedlar possuem fácil manipulação e baixo preço, porém pode haver perdas através do septo, como observado durante o experimento, influenciando a quantificação e identificação dos compostos devido aos problemas de estocagem de uma mistura gasosa volátil. Um menor volume injetado no método de amostragem indireta acarretou uma menor relação percentagem área dos compostos sulfurados em comparação aos outros métodos. A reprodutibilidade da técnica é baixa.

A Figura 2(b) ilustra uma maior relação pico área para os compostos de enxofre quando comparado ao método utilizando a sample bag devido a injeção direta ter ocorrido com volumes constantes e maiores, melhorando a reprodutibilidade da análise.

Figura 3: Comparação dos diferentes métodos de amostragem em termos de percentagem de área

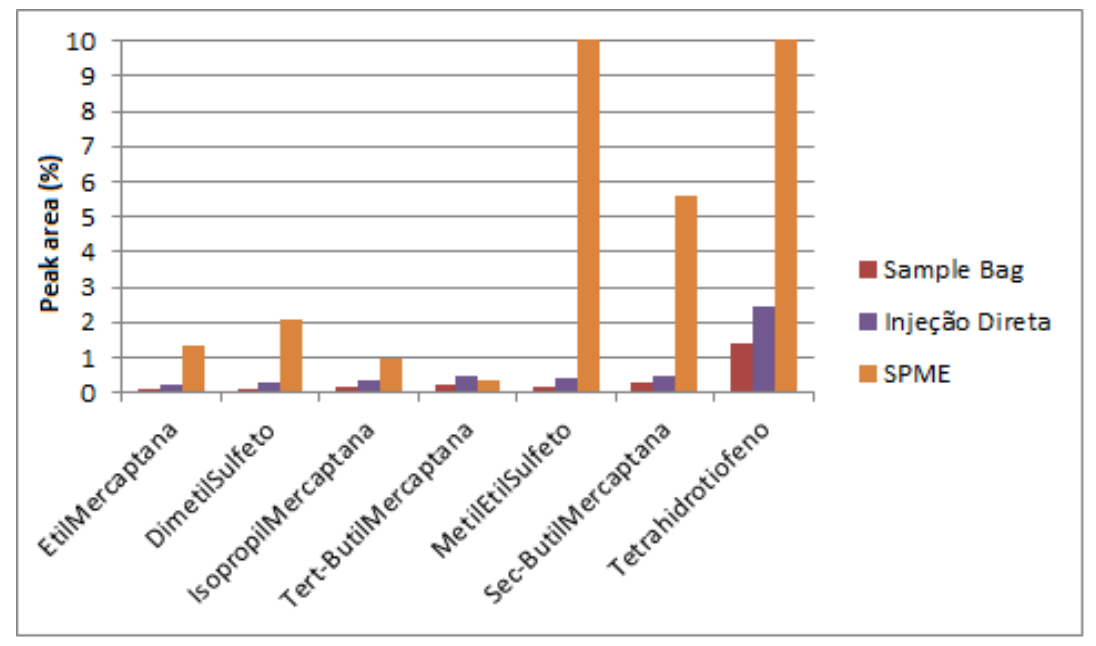

\section{CONCLUSÃO}

Foram realizados 3 diferentes métodos de amostragem: Sample bag, injeção direta e microextração em fase sólida (SPME) para a identificação dos compostos traços de Enxofre em uma matriz de gás natural. A técnica de SPME apresentou o melhor resultado na relação pico área, por se tratar de um método de pré-concentração de analitos, mostrando-se eficiente na detecção dos compostos sulfurados voláteis e de baixas concentrações em cromatografia gasosa. 
Outros fatores como a utilização de uma coluna com maior espessura de filme, as baixas temperaturas de injeção e da coluna, e os maiores volumes injetados da amostra foram importantes para a melhor identificação dos compostos traços sulfurados.

\section{REFERÊNCIAS}

AUGUSTO, C.R. Validação de Metodologia analítica para a Determinação da Composição Química do Gás Natural por Cromatografia em Fase Gasosa Aliada à Estimativa dos Parâmetros de Precisão a Através de Comparação Laboratorial. Tese de Doutorado UFRJ, Programa de Pós Graduação em Tecnologia de Processos Químicos e Bioquímicos, 2011.

CRUZ, L.P.S; CAMPOS, V.P. Sampling and analytical methods for atmospheric reduced sulphur compounds. Quím. Nova, v.31, no.5 São Paulo, 2008.

GRAS,R.; LUONG, J.; CARTER, V.; SIEBEN, L.; CORTES, H. Practical method for the measurement of Alkyl mercaptans in natural gas by multi-dimensional gas chromatography, capillary flow technology, and flame ionization detection. Journal of Chromatography A, 1216, p. 776-2782, 2009

JESUS, C. S.; SANTOS, P. R. M.; SILVA, J. A. S. Dessulfurização do Gás Natural - CCET. Departamento de Engenharia Quimica - UFSE, 2012

KAWASE, K.Y.F. Obtenção, caracterização e aplicação do óleo essencial de orégano. Tese (Doutorado em Ciências). Universidade Federal do Rio de Janeiro- UFRJ, Escola de Química, Curso de Pós Graduação em Tecnologia de Processos Químicos e Bioquímicos, EQ 2013.

MACHADO, M. E. Determinação de compostos orgânicos sulfurados em carvão e petróleo por cromatografia gasosa monodimensional e bidimensional abrangente. Tese de Doutorado UFRGS, Instituto de Química, Programa de Pós Graduação em Química, Porto Alegre - RS, 2011.

NIELSEN, A. T.; JONSSON, S. Quantification of volatile sulfur compounds in complex gaseous matrices by solid-phase microextraction. Journal of Chromatography A, 963, p. 57-64, 2002.

PEDROSO, M.P.; GODOY, L.A.F.; FIDELIS, C.H.V.; FERREIRA, E.C.; POPPI, R.J.; AUGUSTO, F. Cromatografia Gasosa Bidimensional Abrangente $(G C \times G C)$. Instituto de Química, UNICAMP-SP, Brasil. Quím. Nova, Vol. 32, No2, 421- 430, 2009.

SHIMEKIT, B.; MUKHTAR H. Natural Gas Purification Technologies - Major advances for CO2 Separation and future directions. Department of Chemical Engineering. University of technology PETRONAS -Malaysia, 2012.

Os autores agradecem à FAPERJ pelo apoio financeiro. 